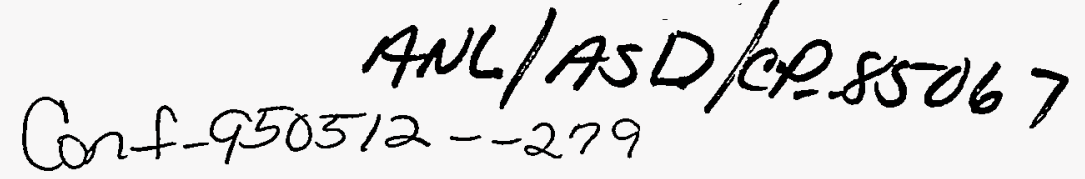

\title{
Local Beam Position Feedback Experiments on the ESRF Storage Ring*
}

\author{
Y. Chung, E. Kahana, J. Kirchman, and A. Lumpkin \\ Argonne National Laboratory, Argonne, IL 60439 \\ J. Meyer, E. Plouviez, K. Scheidt, and E. Taurel \\ European Synchrotron Radiation Facility, F-38043 Grenoble Cedex, France \\ A. Ando, S. Sasaki and A. Taketani \\ SPring-8 Project Team, Kamigori, Hyogo 678-12, Japan
}

The submitted manuscriot has been authorec by a contractor of the U.S. Governmen:

under contract No. W-31-109ENG-3E. under contract No. W-31-109ENG-3E. nonexclusive, royalty-free license to publisr or reproduce the published form of this contribution, or allow others to do so, for U.S. Government purposes.

\section{Abstract}

This paper describes the results of local beam position feedback experiments conducted on the ESRF storage ring using digital signal processing (DSP) under the trilateral agreement of collaboration among ESRF, APS, and SPring-8. Two If beam position monitors (BPMs) in the upstream and downstream of the insertion device (ID) and two $x$-ray BPMs in the sixth cell were used to monitor the electron beam and the $x$-ray beam emitted from the $\mathrm{DD}$, respectively. The local bump coefficients were obtained using the technique of singular value decomposition (SVD) on the global response matrix for the bump magnets and all the available BPMs outside the local bump. The local response matrix was then obtained between the two three-magnet bumps and the position monitors. The data sampling frequency was $4 \mathrm{kHz}$ and a proportional, integral, and derivative (PID) controller was used. The result indicates the closed-loop feedback bandwidth close to $100 \mathrm{~Hz}$ and clear attenuation $(\approx-40 \mathrm{~dB})$ of the $7-\mathrm{Hz}$ beam motion due to girder vibration resonance. Comparison of the results using the rf BPMs and $x$-ray BPMs will be also discussed.

\section{INTRODUCTION}

One of the primary requirements from today's synchrotron light source users is the stringent transverse stability of the $x$ ray beam emitted from the bending magnets (BMs) and insertion devices (IDs). Correction bandwidth exceeding 100 $\mathrm{Hz}$ and long-term drift of less than $10 \%$ of the transverse beam size will be routinely expected from the third-generation synchrotron light sources which are characterized by low emittance of the charged particle beam and high brightness of the photon beam.

Sources of beam motion include ground vibration, mechanical vibration of the accelerator subcomponents, thermal effects, and so forth. In order to counteract the effect of these sources, feedback systems that comprise the beam position monitors (BPMs), corrector magnets, and processing units are typically used [1-4]. The beam position feedback systems can largely be divided into global and local feedback systems according to the extent of correction. and DC and AC

*Work supported by the U.S. Department of Energy, Office of Basic Energy Sciences, under Contract No. W-31-109-ENG-38. feedback systems according to the bandwidth of correction.

In this paper, we will present the results of local AC beam position feedback experiments conducted on the storage ring of the European Synchrotron Radiation Facility (ESRF). The beam position monitor systems consist of two if BPMs in the straight section for an insertion device (ID) and two $x$-ray BPMs monitoring the transverse positions of the $x$-ray beam emanating from the $\mathrm{ID}$. The feedback control is based on digital signal processing (DSP) with the proportional, integral, and derivative (PID) control algorithm. The local bump coefficients are obtained using the technique of singular value decomposition (SVD) of the response matrix for the local correctors and the BPMs outside the bump.

The rest of this paper will consist of a theoretical review of the local beam position feedback with DSP in Section II, description of the experimental setup in Section III, and presentation of the results in Section IV.

\section{THEORY}

Figure 1 shows the schematic of the local beam position feedback using four corrector magnets for control of both the displacement and angle of the $\mathrm{x}$-ray beam. With two degrees of freedom, this can be done by controlling the positions of the charged particle beam at two locations inside the local bump or of the $x$-ray beam at two locations in the downstream of the $x$-ray beamline. The transform between the beam position pair and beam displacement and angle is straightforward. In this work, we do feedback on beam position pairs in the horizontal and vertical planes.

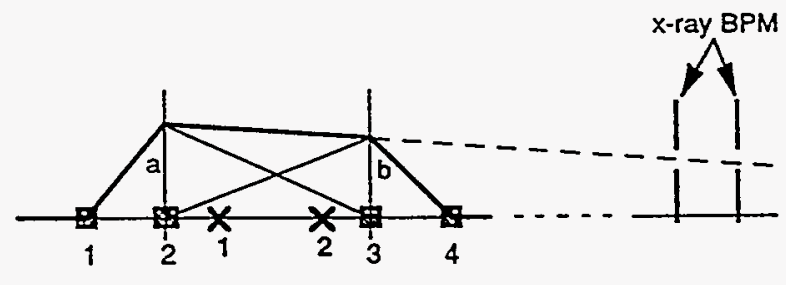

$$
\begin{aligned}
& X \text { : f BPMs } \\
& \text { : Bump magnets } \\
& - \text { : Closed orbit with bump magnets off } \\
& -\quad \text { : New closed orbit with bump magnets on } \\
& \text { : } x \text {-ray radiation }
\end{aligned}
$$

Fig. 1: Schematic of the local beam position feedback using four corrector magnets. 


\section{DISCLAIMER}

Portions of this document may be illegible in electronic image products. Images are produced from the best available original document. 


\section{A, Derivation of the Local Bump Coefficients}

A four-magnet local bump consists of two independent three-magnet bumps $a$ and $b$ as shown in Fig. 1. The correctors in the bumps must be powered with certain ratios dictated by the lattice functions in order to ensure the locality of the bump. Let $\theta_{1}, \theta_{2}, \theta_{3}$, and $\theta_{4}$ be the kick strength on the four correctors, then we have

$$
\boldsymbol{\theta}=\left(\begin{array}{l}
\theta_{1} \\
\theta_{2} \\
\theta_{3} \\
\theta_{4}
\end{array}\right)=\mathbf{K} \cdot\left(\begin{array}{l}
\theta_{a} \\
\theta_{b}
\end{array}\right)=\left(\begin{array}{cc}
K_{1 a} & 0 \\
K_{2 a} & K_{2 b} \\
K_{3 a} & K_{3 b} \\
0 & K_{4 b}
\end{array}\right) \cdot\left(\begin{array}{l}
\theta_{a} \\
\theta_{b}
\end{array}\right),
$$

where $\theta_{a}$ and $\theta_{b}$ represent the strength of the $a$ and $b$ bumps, respectively. The beam positions $\left(x_{1}, x_{2}\right)$ are then related to the bump strengths $\left(\theta_{a}, \theta_{b}\right)$ through the $2 \times 2$ local response matrix $\mathbf{R}_{l}$ as

$$
\left(\begin{array}{l}
x_{1} \\
x_{2}
\end{array}\right)=\mathbf{R}_{l} \cdot\left(\begin{array}{l}
\theta_{a} \\
\theta_{b}
\end{array}\right) .
$$

The $\mathbf{K}$ matrix in Eq. (1) must satisfy the relation $\mathbf{R}_{g l} \cdot \mathbf{K}=\mathbf{0}$, where $\mathbf{R}_{g l}$ is the response of the global orbit outside the local bump to the bump magnets. The elements are called the local bump coefficients. These coefficients can be expressed in terms of the lattice functions $\beta$ and $\psi$ of the correctors [5]. Realistically, other factors such as power supply and magnet efficiencies need to be taken into account as well. To simplify the process, an empirical method was employed to determine the coefficients as explained below.

Let us consider the $M \times 3$ response matrix $\mathbf{R}$ for the correctors in a three-magnet bump and the M BPMs outside the bump. The elements are derived from the expression

$$
R_{i j}=k_{i} k_{c j} \frac{\sqrt{\beta_{i} \beta_{c j}}}{2 \sin (\pi v)} \cos \left(\pi v-\left|\psi_{i}-\psi_{c j}\right|\right),
$$

where $v$ is the tune and $k_{i}$ and $k_{c j}$ are the coefficients of sensitivity for the BPMs and correctors, respectively. Using the technique of singular value decomposition (SVD) $[6,7], \mathbf{R}$ is written as

$$
\mathbf{R}=\mathbf{U} \cdot \mathbf{W} \cdot \mathbf{V}^{\mathrm{T}},
$$

where $\mathrm{U}$ is an $\mathrm{M} \times \mathrm{M}$ unitary matrix, $\mathrm{W}$ is an $\mathrm{M} \times 3$ matrix with off-diagonal elements equal to zero, and $\mathrm{V}$ is a $3 \times 3$ unitary matrix. Using Eq. (3), it can be shown that the determinant of the matrix product $\mathbf{R}^{\mathrm{T}} \cdot \mathbf{R}$ is identical to zero. Therefore, at least one singular value in $W$ should vanish and the corresponding column vector in the matrix $\mathrm{V}$ contains the local bump coefficients. The same procedure is repeated for the other local bump and the K matrix in Eq. (1) is obtained.

The above procedure was tried in the case of the Advanced Photon Source (APS) storage ring with 360 BPMs. The largest response matrix element is approximately 10 $\mathrm{mm} / \mathrm{mrad}$. A random error within $\pm 0.1 \mathrm{~mm} / \mathrm{mrad}$ was introduced, and the local bump coefficients were obtained with different numbers of BPMs between 3 and 358, excluding the two BPMs inside the ID local bump. Figure 2 shows the error in the local bump coefficients and the local bump closure expressed as global orbit distortion in $\mathrm{mm}$ per mrad of bump strength $\left(\theta_{a}\right.$ or $\left.\theta_{b}\right)$.

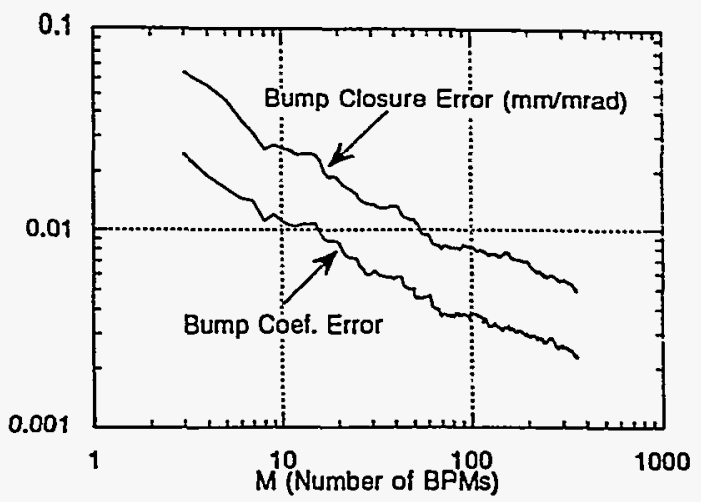

Fig. 2: Application of the SVD-based empirical procedure to the APS storage ring to obtain the local bump coefficients.

\section{B. Digital Signal Processing (DSP)}

Schematic of the feedback signal processing for local beam position feedback is shown in Fig. 3. The set points or the references are $\left(s_{1}, s_{2}\right)$ and the beam positions obtained from the two BPMs are $\left(x_{1}, x_{2}\right)$. The difference signals $\left(s_{1}-x_{1}, s_{2}-x_{2}\right)$ are passed through the low-pass filters (LPF) and the PID controllers. The DSP computes the corrector strengths $\theta_{j}(1 \leq$ $j \leq 4)$ by multiplying the matrix product $K \cdot \mathbf{R}_{l}^{-1}$ by the output of the PID controllers. Since $K_{\text {inv }} \cdot K=1$, the machine response represented by the matrix product $\mathbf{R}_{r} \mathbf{K}_{\mathrm{inv}}$ is such that the resulting beam position is equal to the output of the PID controllers. This renders the local beam position feedback into two independent feedback channels for the BPMs.

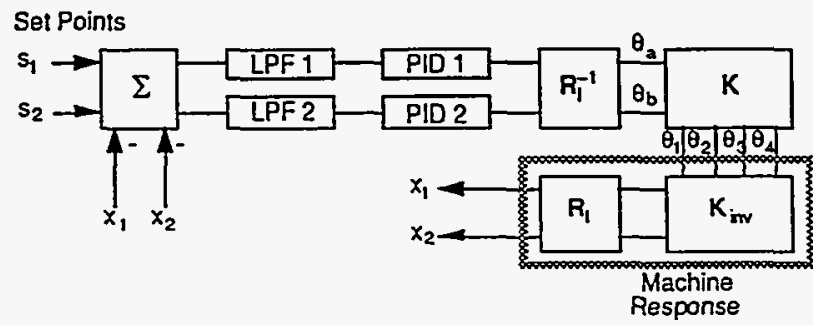

Fig. 3: Schematic of feedback signal processing.

\section{EXPERIMENTAL SETUP}

The If BPMs have button-type pickups, which are connected to filter-comparators (FCs) in the tunnel through short cables. The outputs of the FCs, $\Delta_{x}, \Delta_{y}$ and $\Sigma$. are sent to the monopulse receivers in the signal conditioning and digitizing unit (SCDU) housed in the VXI crate. The data is sent to the DSP in the VME crate through the MXI bus. DSP code development, control of operation, and data acquisition is done using a workstation and a PC connected through ethernet. The $x$-ray BPM analog data is proportional to the 
beam position with sensitivity of $10 \mathrm{~V} / \mathrm{mm}$. This data is digitized by an ADC/DAC board with 12-bit resolution.

The sampling frequency, or number of feedback loops executed per second, was set to $4 \mathrm{kHz}$. Different low-pass filter (LPF) bandwidth PID controller gains were tried to find the optimal point of operation.

\section{RESULTS}

(a)

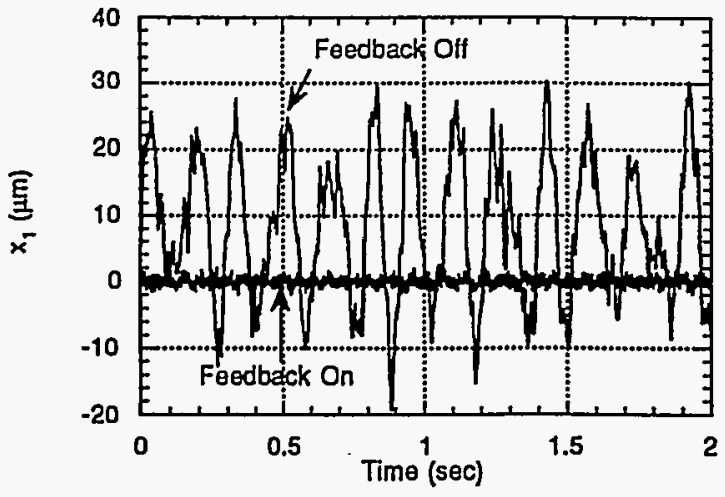

(b)

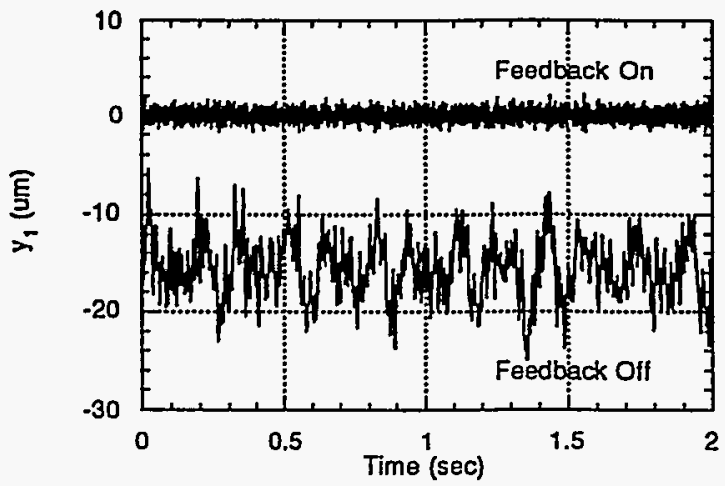

Fig. 4:. Beam motion detected by the If BPMs with feedback on and off in (a) the horizontal plane and (b) the vertical plane. The sampling frequency was $4 \mathrm{kHz}$, LPF bandwidth was $20 \mathrm{~Hz}$, and the $\mathrm{PID}$ gains were: $\mathrm{K}_{\mathrm{p}}=30, \mathrm{~K}_{\mathrm{i}}=0.3, \mathrm{~K}_{\mathrm{d}}=0$.

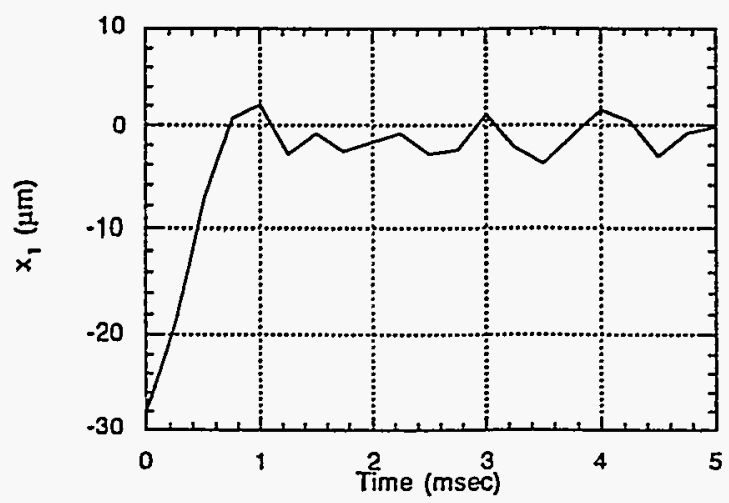

Fig. 5: Impulse response of the feedback system. The rise time is about $600 \mu \mathrm{s}$. The sampling frequency was $4 \mathrm{kHz}$, LPF bandwidth was $20 \mathrm{~Hz}$, and the PID gains were: $K_{p}=20$, $\mathrm{K}_{\mathrm{i}}=0.3, \mathrm{~K}_{\mathrm{d}}=0$.
Figure 4 shows attenuation of the beam motion in the (a) horizontal and (b) vertical planes. The dominant 7- $\mathrm{Hz}$ motion is believed to be due to the ground vibration combined with girder resonance. This beam motion has been successfully arrested by the feedback control. The impulse response of the feedback system shown in Fig. 5 shows a rise time of approximately $500 \mu \mathrm{s}$. We used 4-kHz sampling frequency; $20-\mathrm{Hz}$ LPF bandwidth; and the PID gains $K_{\mathrm{p}}=20, \mathrm{~K}_{\mathrm{i}}=0.3$, and $\mathrm{K}_{\mathrm{d}}=0$. The frequency response of the feedback system in the frequency range $0.1-1000 \mathrm{~Hz}$ is shown in Fig. 6. The bumps near $10 \mathrm{~Hz}$ are due to the measurement fluctuation.

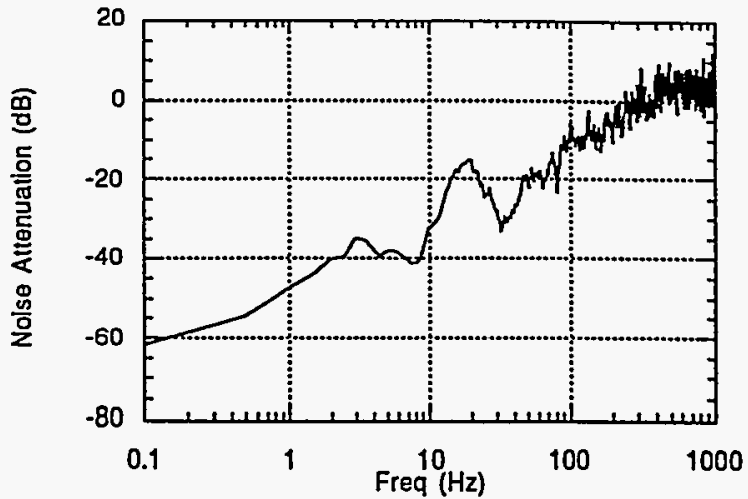

Fig. 6: The frequency response of the feedback system. The feedback parameters are the same as in Fig. 5. The bumps near $10 \mathrm{~Hz}$ are due to measurement fluctuations.

\section{REFERENCES}

[1] R. O. Hettel, "Beam Steering at the Stanford Synchrotron Radiation Laboratory," IEEE Trans. Nucl. Sci., NS-30, No. 4, p. 2228, 1983.

[2] R. J. Nawrocky et al., "Automatic Beam Steering in the NSLS Storage Rings Using Closed Orbit Feedback," Nucl.Instr. and Meth., A266, p. 164, 1988.

[3] Y. Chung, et al., "Closed Orbit Feedback with Digital Signal Processing," Proceedings of the 1994 European Particle Accelerator Conference, London, U.K., p. 1592, 1994.

[4] Y. Chung, et al., "Implementation Status of the Global and Local Beam Position Feedback Systems for the Advanced Photon Source Storage Ring." these proceedings.

[5] S. Krinsky, et al., "Storage Ring Development at the National Synchrotron Light Source." BNL-46615. p. 58. 1991.

[6] G. H. Golub and C. Reinsch. "Singular Value Decomposition and Least Squares Solutions," Numer. Math. 14, pp. 403-420, 1970, and references therein. Also in J. H. Wilkinson and C. Reinsch, Linear Algebra. vol. II of Handbook for Automatic Computation (Springer-Verlag, New York, 1971).

[7] Y. Chung, G. Decker and K. Evans, Jr., "Closed Orbit Correction Using Singular Value Decomposition of the Response Matrix." Proceedings of the 1993 IEEE Particle Accelerator Conference, Washington. D.C., p. 2263, 1993. 


\section{DISCLAIMER}

This report was prepared as an account of work sponsored by an agency of the United States Government. Neither the United States Government, nor any agency thereof, nor any of their employees, makes any warranty, express or implied, or assumes any legal liability or responsibility for the accuracy, completeness, or usefulness of any information, apparatus, product, or process disclosed, or represents that its use would not infringe privately owned rights. Reference herein to any specific commercial product, process, or service by trade name, trademark, manufacturer, or otherwise does not necessarily constitute or imply its endorsement, recommendation, or favoring by the United States Government or any agency thereof. The views and opinions of authors expressed herein do not necessarily state or reflect those of the United States Government or any agency thereof. 